\title{
Many worlds in one
}

\author{
Jaume Garriga \\ IFAE, Departament de Física, Universitat Autonoma de Barcelona, 08193 Bellaterra, Barcelona, Spain \\ and Institute of Cosmology, Department of Physics and Astronomy, Tufts University, Medford, Massachusetts 02155
}

Alexander Vilenkin

Institute of Cosmology, Department of Physics and Astronomy, Tufts University, Medford, Massachusetts 02155

(Received 16 February 2001; published 26 July 2001)

\begin{abstract}
A generic prediction of inflation is that the thermalized region we inhabit is spatially infinite. Thus, it contains an infinite number of regions of the same size as our observable universe, which we shall denote as $\mathcal{O}$ regions. We argue that the number of possible histories which may take place inside of an $\mathcal{O}$ region, from the time of recombination up to the present time, is finite. Hence, there are an infinite number of $\mathcal{O}$ regions with identical histories up to the present, but which need not be identical in the future. Moreover, all histories which are not forbidden by conservation laws will occur in a finite fraction of all $\mathcal{O}$ regions. The ensemble of $\mathcal{O}$ regions is reminiscent of the ensemble of universes in the many-world picture of quantum mechanics. An important difference, however, is that other $\mathcal{O}$ regions are unquestionably real.
\end{abstract}

DOI: 10.1103/PhysRevD.64.043511

\section{INTRODUCTION}

The presently observable spacetime region of the universe is defined by the interior of our past light cone. It extends back to the time of recombination $t_{\text {rec }}$ beyond which the universe is opaque to electromagnetic waves. For brevity, we shall denote as our $\mathcal{O}$ region the interior of this past light cone from the time of recombination up to the present. At time $t_{r e c}$ this region was nearly featureless with tiny deviations from homogeneity, presumably caused by quantum fluctuations during inflation. It was practically indistinguishable from same-size regions in other parts of the universe.

At later times, the small initial fluctuations were amplified by gravitational instability, and the properties of $\mathcal{O}$ regions began to diverge. By the time of structure formation, the details of galaxy distribution in different $\mathcal{O}$ regions varied considerably (although statistically the regions were very similar). Later on, the evolution of life and intelligence could be influenced by statistical and quantum fluctuations, leading to further divergence of properties. The histories of different $\mathcal{O}$ regions are thus expected to be rather different.

It is conceivable, however, that there will be some regions with identical histories. Whether or not this situation is to be expected depends on the relative number of $\mathcal{O}$ regions and of the possible histories. In this paper, we are going to argue that the number of distinct histories in an $\mathcal{O}$ region is finite, while the number of $\mathcal{O}$ regions in the universe is infinite, and thus there should be an infinite number of other regions with histories identical to ours. Moreover, all histories which are not strictly forbidden by conservation laws occur in a finite fraction of the $\mathcal{O}$ regions. The argument below is far from being a rigorous proof of these statements, but we do believe that it makes them rather plausible.

\section{THE NUMBER OF POSSIBLE HISTORIES IS FINITE}

It is relatively straightforward to argue that the total number of "subjective" histories which may take place in a given $\mathcal{O}$ region is finite. For this purpose, we may consider a set of
PACS number(s): 98.80.Hw, 98.80.Cq

"subjects" consisting of what Gell-Mann and Hartle [1] have termed IGUSes: information gathering and utilizing systems (humans are just a particular example of IGUSes). The number of IGUSes which will develop in an $\mathcal{O}$ region is finite, since these IGUSes have to be built from finite resources. If the "minds" of IGUSes can be modeled as discrete computers, which operate much like our own brain by discrete firings of neurons, then we may define the subjective history of the $\mathcal{O}$ region as the sequence of all operations performed by all of the minds contained in the $\mathcal{O}$ region. Since the speed of computation is finite and the available time is finite, the number of subjective histories will also be finite.

In this section, however, we shall argue for the stronger statement that even the number of objective histories is finite. If the universe obeyed the laws of classical physics, then the history of an $\mathcal{O}$ region would be completely determined by the initial conditions at $t_{r e c}$. In quantum mechanics, all histories that are consistent with exact conservation laws can occur with some probability, and in this sense the number of possibilities is greatly increased. On the other hand, a classical description can be made arbitrarily detailed, hence the number of possible classical histories is infinite. However, in quantum mechanics there is a limit to the level of detail that can be achieved.

Modern formulations of quantum theory are given in terms of alternative, decoherent, coarse-grained histories of the system [1-3] (for a review and further references, see Ref. [4]). To specify a coarse-grained history, one can partition the spacetime region into cells and divide the possible values of physical quantities into finite bins. A history is specified by indicating the appropriate bins for the average values of all quantities in each of the cells. A history is a semiclassical concept, and it has been argued [1,5] that the relevant quantities for a semiclassical description are "hydrodymamic" variables, such as energy, momentum, and various conserved and approximately conserved charges. The histories can be regarded as distinct if they decohere, that is, if the decoherence functional $D(A, B)$ satisfies 
$|D(A, B)|^{2}<\epsilon|D(A, A) D(B, B)|$ for any two histories $A$ and $B$ in the set of all possible histories. If this condition is satisfied with a sufficiently small $\epsilon \ll 1$, then a probability can be assigned to each history in the set. The sizes of the cells and of the bins for the values of the physical quantities cannot be made arbitrarily small, since otherwise the property of decoherence would be destroyed. The best one can do is to obtain a maximally refined set of histories, such that further refinement would make interference between histories nonnegligible.

For a system of a finite size observed for a finite interval of time, the total number of cells is finite. If the possible values of all physical quantities are bounded, then the number of bins for each quantity is finite and it is clear that the total number of distinct histories is also finite.

In the absence of gravity, the boundedness of physical quantities usually follows from the finiteness of the energy of the system. The gravitational energy is not positive definite, and in the presence of gravity the matter energy density can get arbitrarily large when it is compensated by equally large negative gravitational energy density. This happens, for example, during gravitational collapse in the interiors of black holes, in a recollapsing closed universe, and could happen in some extremely large quantum fluctuations. These extreme situations, however, are far removed from the low-energy world of the human experience, and it seems reasonable to isolate them when comparing the histories of different $\mathcal{O}$ regions. One could, for example, assign all values of physical quantities corresponding to over-Planckian energy density of matter to a single bin. Then, histories that differ only in spacetime regions of higher than Planckian density would be regarded as identical by humans (and presumably by all other IGUSes). ${ }^{1}$ A much lower threshold on the density would of course suffice, but the important point for us here is that the relevant range of variation of the physical quantities is finite.

The identification of two histories which differ only in regions of energy density higher than a certain cutoff has been justified by the fact that they are perceived as equal by IGUSes. This is a somewhat "subjective" identification of histories, in the sense described at the beginning of this section. An alternative line of reasoning which is completely objective, and which indicates that the number of possible histories is finite, is based on the so-called holographic bounds. The original bound, conjectured by 't Hooft [6] and Susskind [7], claims that the entropy in a finite region of space satisfies

$$
S \leqslant A / 4,
$$

\footnotetext{
${ }^{1}$ It is also possible that super-Planckian densities in cells need not be considered because they are excluded by the fundamental theory. If cell sizes are large compared to the Planck length and the density is greater than Planckian, $l \gg 1$ and $\rho>1$ in Planck units, then the corresponding Schwarzschild radius $r_{s} \sim \rho l^{3}$ is much greater than the cell size $l$. One could expect that regions of this kind would be hidden from external observers by event horizons.
}

where $A$ is the area (in Planck units) of a surface surrounding the region. This bound is related to the entropy bounds introduced by Bekenstein [8]. As it stands, the bound (1) cannot be true in general, since it is known to be violated for collapsing regions inside their Schwarzschild radius and for sufficiently large spherical regions of an expanding universe. Bousso [9] suggested a modification of the bound which may be free from these limitations. The precise form of the bound will not be important for our discussion here. We shall simply assume that the entropy in a finite region cannot be arbitrarily large,

$$
S \leqslant \ln N
$$

where $N$ may depend on the size of the region. Since $\mathcal{O}$ regions are finite, they should also obey the bound.

The bound (2) implies that the number of linearly independent quantum states for an $\mathcal{O}$ region does not exceed $N$. In other words, the dimension of the corresponding Hilbert space is $d \leqslant N$. Thus, any complete set of projection operators characterizing the configuration of the system at any given moment of time is finite. A coarse-grained history can be specified by giving a complete set of coarse-grained projection operators at discrete intervals of time. Thus, if the number of time steps is finite, the number of coarse-grained histories will also be finite.

Finally, let us estimate the number of possible histories of an $\mathcal{O}$ region using the method of cells and bins. If $N_{c}$ is the number of spacetime cells and $N_{b}$ is the number of relevant bins in field space, the number of coarse-grained histories $N_{h}$ is given by

$$
\ln N_{h} \sim N_{c} \ln N_{b}
$$

The spacetime volume inside of our past light cone from the time of recombination is of order $t_{0}^{4} \sim 10^{244} t_{P}$, where $t_{0}$ $\sim 10^{10} \mathrm{yr}$ is the age of the universe and $t_{P} \sim 10^{-44} \mathrm{~s}$ is the Planck time. The number of spacetime cells of size $L$ is thus given by

$$
N_{c} \sim 10^{244}\left(L M_{P}\right)^{-4} .
$$

To estimate the number of bins, consider for instance a scalar field $\phi$. The equal time field commutator is $\left[\phi(x), \dot{\phi}\left(x^{\prime}\right)\right]$ $=i \delta\left(x-x^{\prime}\right)$. Integrating over $x$ and $x^{\prime}$ over the volume $V$ $=L^{3}$ and dividing over $V^{2}$ gives

$$
[\Phi, \dot{\Phi}]=i / V
$$

where $\Phi$ is the smeared field (averaged over the cell). The uncertainty relation following from the last equation is

$$
(\Delta \Phi)(\Delta \dot{\Phi}) \sim 1 / V .
$$

If we try to specify $\Phi$ with an accuracy better than $1 / L$, then the uncertainty in $\dot{\Phi}$ is greater than $1 / L^{2}$, which means that the variation of $\Phi$ over the period $L$ is greater than $1 / L$. Thus, a lower bound on the size of the bins which can be used to specify a semiclassical history is given by $1 / L$, and the number of bins will be bounded by

$$
N_{b} \lesssim \phi_{\max } L,
$$


where $\phi_{\max }$ is an estimate of the allowed range of the field variable. The dependence of the number of bins on $L$ is only linear, and the number of histories $N_{h}$ will be maximized for the lowest possible value of $L$ compatible with more than one bin. In usual particle physics models $\phi_{\max } \leqslant M_{P}$, and in this case the number of coarse grained histories for the scalar field is bounded by

$$
\ln N_{h} \lesssim 10^{244} .
$$

The range of the field could in principle be much larger than Planckian. For instance, an exact Goldstone boson could have an infinite range. In this case, the value of $\phi_{\max }$ to be used above is still finite. Requiring that the present average kinetic energy density be lower than, say, the Planck energy density we have $\phi_{\max } \lesssim t_{0} M_{P}^{2} \sim 10^{61} M_{P}$. When substituted in Eq. (3), and assuming that the minimum size of a cell is the Planck length, this would increase the exponent in Eq. (4) by a couple of units.

The standard model allows for many observables other than canonical scalar fields. As mentioned above, it is not clear how many of these observables should be used to specify coarse-grained histories and to calculate the number of bins. We may take the point of view that semiclassical coarse-grained histories are fully specified by the average values of the electromagnetic field strength, the different baryonic and leptonic charge densities and the gravitational field in each cell [1]. One might also consider the local values of, say, the Higgs field and of the non-Abelian gauge bosons, but nothing essential seems to be lost from the classical point of view if all possible values of these fields are "traced over" in a single bin. Therefore, the total number of relevant observables seems to be very moderate. The size of the cells which is necessary to ensure decoherence is likely to be much larger than the Planck length, so the upper bound (4) grossly overestimates the number of decoherent histories. A more realistic estimate may perhaps be obtained by considering cells of atomic size $L \sim 10^{-8} \mathrm{~cm}$. This would cut the exponent in Eq. (4) down to less than 150.

\section{THE NUMBER OF $\mathcal{O}$ REGIONS IS INFINITE}

We shall now argue, in the framework of inflationary cosmology, that the number of $\mathcal{O}$ regions in the universe is infinite.

One of the striking aspects of inflation is that, generically, it never ends (for a review of inflation, see e.g. Ref. [10]). The evolution of the inflaton field $\phi$ is influenced by quantum fluctuations, and as a result thermalization does not occur simultaneously in different parts of the universe. On small scales, the fluctuations in the thermalization time give rise to a spectrum of small density fluctuations, but on large scales they make the universe extremely inhomogeneous. In most of the models, one finds that, at any time, there are parts of the universe that are still inflating and that the total volume of the inflating regions is growing with time $[11,12]$. This picture is often referred to as "stochastic inflation" or "eternal inflation."

The spacetime structure of an eternally inflating universe is illustrated in Fig. 1 [13]. The vertical axis is time and the

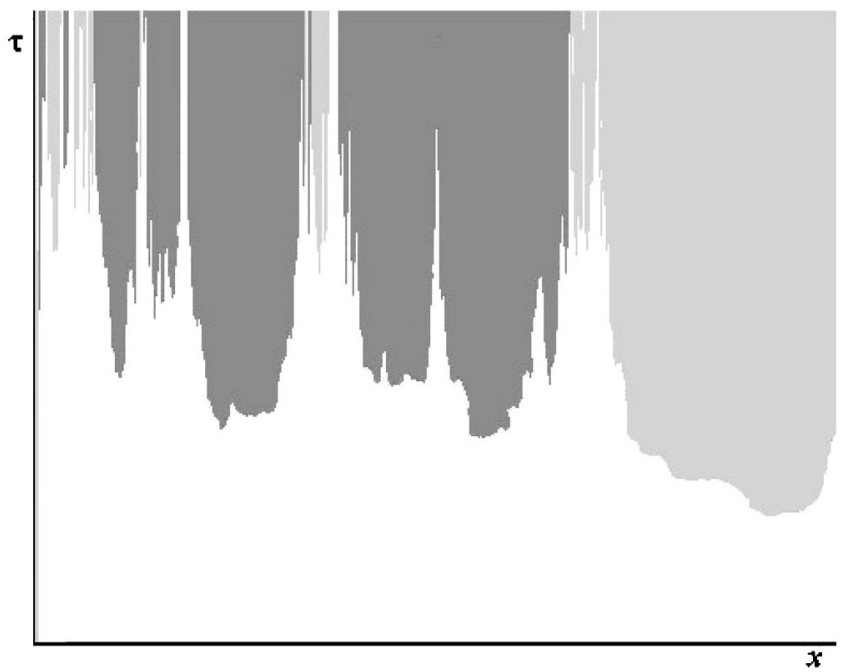

FIG. 1. A numerical simulation of the spacetime structure of an inflating universe [13]. The simulation corresponds to a double-well inflaton potential, with two degenerate minima where the inflaton takes the values $\pm \eta$. Inflating regions are white, while thermalized regions with inflaton values equal to $+\eta$ and $-\eta$ are shown with different shades of gray.

horizontal axis is the comoving distance in one of the spatial directions. The boundaries between inflating and thermalized regions play the role of the big bang for the corresponding thermalized regions. In the figure, these boundaries become nearly vertical at late times, so that it appears that they become timelike. The reason is that the horizontal axis is the comoving distance, with the expansion of the universe factored out. The physical distance is obtained by multiplying by the expansion factor $a(t)$, which grows exponentially as we go up the time axis. If we used the physical distance in the figure, the thermalization boundaries would "open up" and become nearly horizontal (but then it would be difficult to fit more than one thermalized region in the figure).

The thermalization boundaries are surfaces of constant energy density of matter. It can be shown that they are infinite spacelike surfaces and, with an appropriate choice of coordinates, each thermalized region is an infinite subuniverse containing an infinite number of galaxies. These infinite sub-universes can fit into finite comoving regions of the universe due to the exponential expansion of the surrounding inflating regions.

The spacetime structure of a thermalized region near the thermalization boundary is illustrated in Fig. 2. The thermalization is followed by hot radiation era and then by a matter dominated era during which luminous galaxies are formed and civilizations flourish. All stars eventually die, and thermalized regions become dark, cold, and probably not suitable for life. Hence, observers are to be found within a layer of finite (temporal) width along the thermalization boundaries in Fig. 1. The tips of the light cones of the $\mathcal{O}$ regions lie within that layer. Since $\mathcal{O}$ regions have a finite size, it is easily seen that the number of non-overlapping $\mathcal{O}$ regions in each thermalized region is infinite.

Do histories unfolding in different $\mathcal{O}$ regions do so simultaneously, or do they belong to different epochs? The answer 


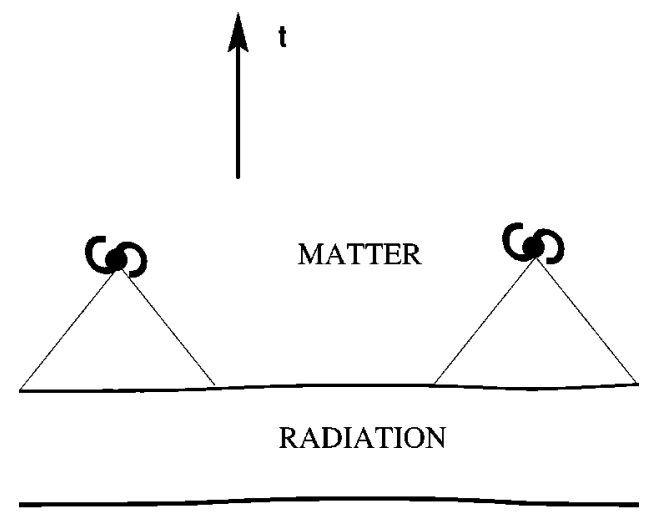

INFLATION

FIG. 2. Spacetime structure near the thermalization surface. After inflation, a period of radiation domination is followed by the matter dominated era, where galaxies form and civilizations flourish. Represented in the figure are the $\mathcal{O}$ regions of two observers who live in mutually very distant galaxies.

to this depends on one's choice of the time coordinate. The time coordinate in Fig. 1 is the proper time along the worldlines of a congruence of comoving observers. With this choice, the constant time surfaces contain inflating regions, as well as thermalized regions at different stages of their evolution, from the high-energy state at thermalization to extreme low densities and temperatures after the death of stars. A more natural choice in the interiors of thermalized regions is to use the surfaces of constant energy density as constant time surfaces. (Such surfaces are Cauchy surfaces for the corresponding thermalized regions, but not for the rest of the universe.) Then each thermalized region is described as an infinite sub-universe, with all of its $\mathcal{O}$ regions evolving simultaneously.

\section{SOME IMPLICATIONS}

We have argued that (i) the number of possible histories for an $\mathcal{O}$ region is finite and (ii) the number of $\mathcal{O}$ regions in each of the thermalized regions of the universe is infinite. With an appropriate choice of the time coordinate, all $\mathcal{O}$ regions in a given thermalized region originate at the same time $\left(t=t_{\text {rec }}\right)$ and their histories are unfolding, so to say, as we speak.

The initial state of each $\mathcal{O}$ region can be characterized by a density matrix, and the probabilities for all possible histories can in principle be determined following the usual rules of quantum mechanics. ${ }^{2}$ All histories consistent with exact conservation laws will have non-vanishing probabilities and will occur in an infinite number of $\mathcal{O}$ regions. It follows that there should be an infinite number of $\mathcal{O}$ regions whose history is identical to ours.

\footnotetext{
${ }^{2}$ We expect the histories in different $\mathcal{O}$ regions to be statistically independent. For nearby regions, there will be some correlations in the low multipoles of the microwave sky, but such correlations can be avoided by selecting an ensemble of well-separated regions.
}

This picture is reminiscent of the many-world interpretation of quantum mechanics [14-16]. According to this interpretation, the wave function of the universe describes a multitude of disconnected universes with all possible histories. The reality of the other universes is still a matter of controversy (see, e.g., Ref. [17]), but the issue is rather academic, since the existence of these universes cannot be tested in any direct way.

The ensemble of $\mathcal{O}$ regions that we discussed in this paper is very similar to the ensemble of universes in the manyworld picture. An important difference, however, is that other $\mathcal{O}$ regions are unquestionably real. There are infinitely many of them in our own thermalized region. Nonoverlapping $\mathcal{O}$ regions are causally disconnected, so at present we cannot get any information from other $\mathcal{O}$ regions. However, information exchange will become possible in the future. Given enough time, we might even be able to travel to what used to be other $\mathcal{O}$ regions and to compare their histories with ours. ${ }^{3}$

The existence of $\mathcal{O}$ regions with all possible histories, some of them identical or nearly identical to ours, has some potentially troubling implications. Whenever a thought crosses your mind that some terrible calamity might have happened, you can be assured that it has happened in some of the $\mathcal{O}$ regions. If you nearly escaped an accident, then you were not so lucky in some of the regions with the same prior history. On a positive side, some amusing situations can be entertained where distant copies of ourselves play all sorts of different roles. Some readers will be pleased to know that there are infinitely many $\mathcal{O}$ regions where Al Gore is President and-yes-Elvis is still alive.

Consider the ensemble of $\mathcal{O}$ regions containing a civilization whose history is identical to ours up to the present time (call this ensemble $\mathcal{E}$ ). In the future the paths of these civilizations will of course diverge, spanning all possible histories. Each civilization in $\mathcal{E}$ can be quantitatively characterized by a set of parameters such as its lifetime, its age at the time when it made certain technological advances, the extent to which it colonized the galaxy, etc. One could in principle determine the probability distribution for these parameters. A priori, one expects that our civilization is a typical representative of the ensemble, with most of the parameters near the peak of the distribution. This is an extension of the "principle of mediocrity" [18] (see also Refs. [19] and [20]) to the realm of history.

We conclude with some comments on the future of civilizations in the ensemble $\mathcal{E}$. It appears very unlikely that a civilization can survive forever [21,22]. Even if it avoids natural catastrophes and self-destruction, it will in the end run out of energy. The stars will eventually die, other sources of energy (such as tidal forces) will also come to an end, and

\footnotetext{
${ }^{3} \mathrm{We}$ emphasize that the picture of the universe presented here is independent of the interpretation of quantum mechanics. If the many world interpretation is adopted, then there is an ensemble of eternally inflating universes, each having an infinite number of $\mathcal{O}$ regions. Our picture should apply to each of the universes in the ensemble.
} 
our thermalized region will become cold, dark, and inhospitable to life. This argument, however, is statistical in nature, since it is based on the second law of thermodynamics. We know that entropy can decrease due to thermal fluctuations, and it is in principle possible that such fluctuations can sustain a civilization for an arbitrarily long time. The probability that a civilization will survive on this kind of "fuel" for a time $t$ is of course a sharply decreasing function of $t$. However, for any finite $t$ the probability is finite, and thus an infinite number of civilizations in our thermalized region will live longer than any given time. Needless to say, there is little hope that we are going to be among the lucky civilizations whose life will be prolonged by thermal fluctuations.

If we are doomed to perish, then perhaps we could perpetuate our legacy by sending messages to new thermalized regions in our future? In Ref. [23] we explored some scenarios of this sort, where messages (tightly packed in durable containers) could be sent into new inflating regions spawning from our own thermalized region. A new inflating region within our thermalized region can be created by quantum tunneling or by virtue of strong violations of the weak energy condition (see Ref. [23] and references therein). New civilizations arising after thermalization in the newly created inflating regions might then be able to read those messages. Recipients of our messages could also send messages to the future, and so on. We would then become a branch in an infinite "tree" of civilizations, and our accumulated wisdom would not be completely lost.

However, in view of the discussion above, it seems that sending messages into new inflating regions is a rather useless activity. If the history where our message is received in some $\mathcal{O}$ region is not forbidden by the laws of physics, then we know that it will be repeated infinitely many times in different $\mathcal{O}$ regions of the same thermalization surface. Hence, any finite number of sent messages does not increase the chance of receiving a message [24]. An infinite number of additional messages will come into existence by chance through thermal or quantum fluctuations. Whatever our motivation for sending the message could be, it is rather pointless to do so because an infinite number of messages gets received anyway. ${ }^{4}$

Note Added. After this paper was submitted, we learned about the early work by Ellis and Brundrit [25] who discussed some of the issues we raised here in the context of an open Robertson-Walker universe. By assumption, such a universe is infinite and homogeneous (on average), and thus contains an infinite number of galaxies. If our universe is of this kind, then Ellis and Brundrit argued that there should be some regions in the universe with histories very similar to that in our region. Our analysis here goes beyond that of Ellis and Brundrit in two respects: (i) the infinite number of $\mathcal{O}$ regions in our picture is a generic consequence of inflation and does not have to be independently postulated, and (ii) we argued that the number of distinct histories is finite, which allowed us to conclude that there should be regions with histories identical to ours.

\section{ACKNOWLEDGMENTS}

We are grateful to Ken Olum for interesting discussions and to David Deutsch and Jim Hartle for useful correspondence. This work was supported by the Templeton Foundation under grant COS 253. J.G. is partially supported by CICYT, under grant AEN99-0766, and by the Yamada Foundation. A.V. is partially supported by the National Science Foundation.

\footnotetext{
${ }^{4}$ Of course, not only the message representing our would-be legacy will be spontaneously created. Nonsensical messages will also be created at a much higher rate [24] (perchance resemblingalas-copies of the present paper. IGUSes beware).
}

[1] M. Gell-Mann and J.B. Hartle, in Complexity, Entropy and the Physics of Information, SFI Studies in the Sciences of Complexity, Vol. VIII, edited by W. Zurek (Addison-Wesley, Reading, MA 1990); Phys. Rev. D 47, 3345 (1993).

[2] R.B. Griffiths, Phys. Rev. Lett. 70, 2201 (1993); J. Stat. Phys. 36, 219 (1984).

[3] R. Omnes, J. Stat. Phys. 53, 893 (1988); Rev. Mod. Phys. 64, 339 (1992).

[4] J.B. Hartle, in Les Houches Summer School 1992, p. 285, gr-qc/9304006.

[5] J.J. Halliwell, Phys. Rev. Lett. 83, 2481 (1999).

[6] G. 't Hooft, gr-qc/9310026.

[7] L. Susskind, J. Math. Phys. 36, 6377 (1995).

[8] J.D. Bekenstein, Phys. Rev. D 23, 287 (1981); Phys. Lett. B 481, 339 (2000).

[9] R. Bousso, J. High Energy Phys. 07, 004 (1999); 11, 038 (2000)

[10] A.H. Guth, Phys. Rep. 333-334, 555 (2000).

[11] A. Vilenkin, Phys. Rev. D 27, 2848 (1983).
[12] A.D. Linde, Phys. Lett. B 175, 395 (1986).

[13] V. Vanchurin, A. Vilenkin, and S. Winitzki, Phys. Rev. D 61, 083507 (2000).

[14] H. Everett, Rev. Mod. Phys. 29, 454 (1957).

[15] B. DeWitt, Phys. Today 23 (9), 30 (1970).

[16] D. Deutsch, The Fabric of Reality (Penguin, Torento, 1998).

[17] J.R. Brown and P.C.W. Davies, The Ghost in the Atom (Cambridge University Press, Cambridge, England, 1993).

[18] A. Vilenkin, Phys. Rev. Lett. 74, 846 (1995).

[19] J. Leslie, Bull. Can. Nuc. Soc. 10, 10 (1989); The End of the World (Routledge, London, New York, 1996).

[20] J.R. Gott III, Nature (London) 363, 315 (1993).

[21] F.J. Dyson, Rev. Mod. Phys. 51, 447 (1979).

[22] L.M. Krauss and G.D. Starkman, Astrophys. J. 531, 22 (2000).

[23] J. Garriga, V.F. Mukhanov, K.D. Olum, and A. Vilenkin, Int. J. Theor. Phys. 39, 1887 (2000).

[24] D. Deutsch (private communication).

[25] G.F.R. Ellis and G.B. Brundrit, Q. J. R. Astron. Soc. 20, 37 (1979). 